\title{
Virtual Draw of Tubular Hollow-Core Fibers
}

\author{
Gregory T. Jasion, John R. Hayes, Natalie V. Wheeler, Yong Chen, Thomas D. Bradley, Seyed Reza \\ Sandoghchi, Marco Petrovich, David J. Richardson, Francesco Poletti \\ Optoelectronics Research Centre, University of Southampton, Highfield, Southampton, SO17 1BJ, UK \\ Author e-mail address: g.jasion@soton.ac.uk
}

\begin{abstract}
A numerical model that accurately predicts the fabricated geometry of tubular hollowcore fibers is presented and experimentally validated. Such a model can be invaluable in driving design decisions for yield upscaling and loss reduction.

OCIS codes: (060.0060) Fiber optics and optical communications; (060.2280) Fiber design and fabrication; (060.4005) Microstructured fibers.
\end{abstract}

\section{Introduction}

Hollow core microstructured fibers guide light in a hollow air core making them well suited to low latency telecoms and high power delivery. They confine light by means of a delicate microstructured cladding and broadly fall into two categories: the honeycomb-like cladding photonic bandgap fiber, which makes use of a periodic lattice of nodes, and the anti-resonant/inhibited coupling fiber, which confines light using membranes of a specified thickness. The anti-resonant variety has been fabricated in several forms, initially as Kagomé [1] and more recently as Tubular and Nested Anti-resonant Nodeless Fiber geometries, which have received much attention due to their simplicity, broad bandwidth, and the promise of extremely low transmission losses [2-5]. Tubular fibers typically consist of a thick jacket tube inside of which are attached a number of non-touching thin walled capillaries forming a hollow core between them, Fig. 1.These fibers are drawn from homothetically scaled preforms on a fiber drawing tower. During the draw, the surface tension on the glass-air interfaces causes the capillaries to contract; this is countered by application of gas pressures in the core region and in the capillaries. Precise control of the applied capillary pressure is essential to reduce the gap between neighboring capillaries, crucial for achieving low loss [5].

Here we introduce a numerical method to virtually draw tubular fibers from a given preform, to predict the fiber geometry resulting from any draw condition. We then validate this model against two different experimental fiber draws, and explain how it can assist the fabrication of uniform, high performance fibers with greater yield.

\section{Model methodology}

In tubular fibers the majority of the core-surrounding capillary walls are free and circular. To model their fluid dynamic evolution we use the semi-analytical Fitt model [6] to calculate the neck-down shape for the outer jacket of the fiber, as already adopted in the MSEM [7]. We then extend the model and use the axial strain rate solution from the jacket to solve the same equations for the drawdown of the internal capillaries.

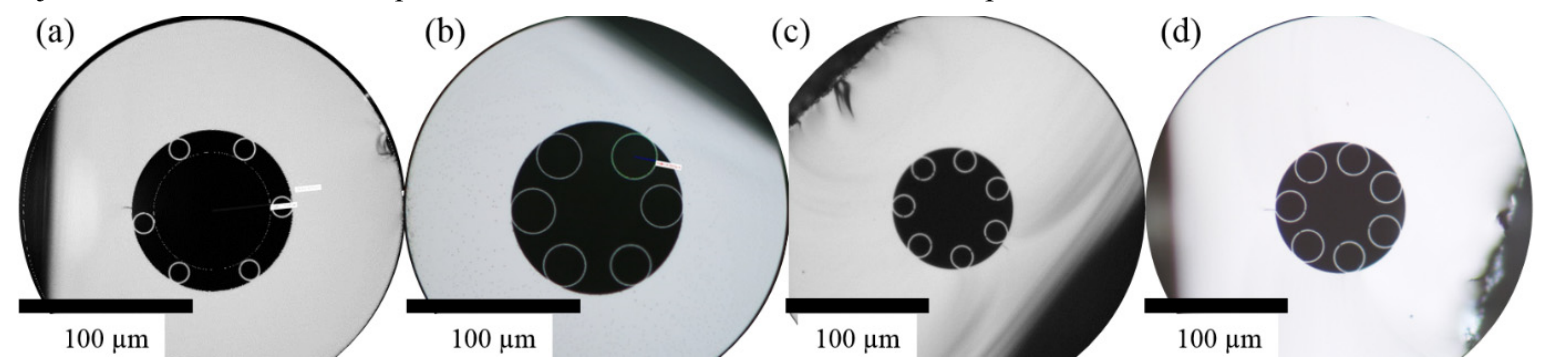

Fig. 1. 6C fibers with low, (a), and high, (b), capillary pressure. 7C fibers with low, (c), and high, (d), capillary pressure.

\section{Fiber fabrication}

To validate the model, two experimental fiber draws were conducted from two different preforms with 6 capillaries (6C) and 7 capillaries (7C). Each fiber draw produced several samples with increasing capillary pressure to expand the capillaries while maintaining the same overall fiber dimensions. Examples of the fibers can be seen in Fig. 1(ad). Lowest loss is achieved when the gap between neighboring capillaries is a minimum provided that they do not come into contact with each other. A differential pressure is applied to the core and either the individual capillaries or the annular region surrounding it. Table 1 summarizes the preform geometry and draw parameters for the two draws. The simulation used the same geometric and draw parameters as the experiment, and furnace conditions were governed by the draw tension which is matched to the experiment [7]. 
Table 1. Preform geometry and draw parameters.

\begin{tabular}{lcc} 
& $6 \mathrm{C}$ & $7 \mathrm{C}$ \\
\hline Cane capillary diameter & $0.46 \mathrm{~mm}$ & $0.52 \mathrm{~mm}$ \\
Cane diameter & $2.9 \mathrm{~mm}$ & $2.4 \mathrm{~mm}$ \\
Jacket tube ID/OD & $3 / 10 \mathrm{~mm}$ & $2.7 / 9.4 \mathrm{~mm}$ \\
Fiber ID/OD & $100 / 220 \mu \mathrm{m}$ & $80 / 255 \mu \mathrm{m}$ \\
Draw tension & $300 \mathrm{~g}$ & $340 \mathrm{~g}$
\end{tabular}

\section{Results and discussion}

The capillary diameters in each fiber sample were measured and their mean value is plotted in Fig. 2. The error bars indicate the min and max diameters measured; solid lines show the simulated results. To match the experiment the simulations required a single calibration point in the form of a constant pressure offset from the experimentally imposed values, $1.35 \mathrm{kPa}$ for $6 \mathrm{C}$ and $1.5 \mathrm{kPa}$ for $7 \mathrm{C}$. This is likely due to unmeasurable (and variable from draw to draw) pressure drops in the preform pressurization chamber.
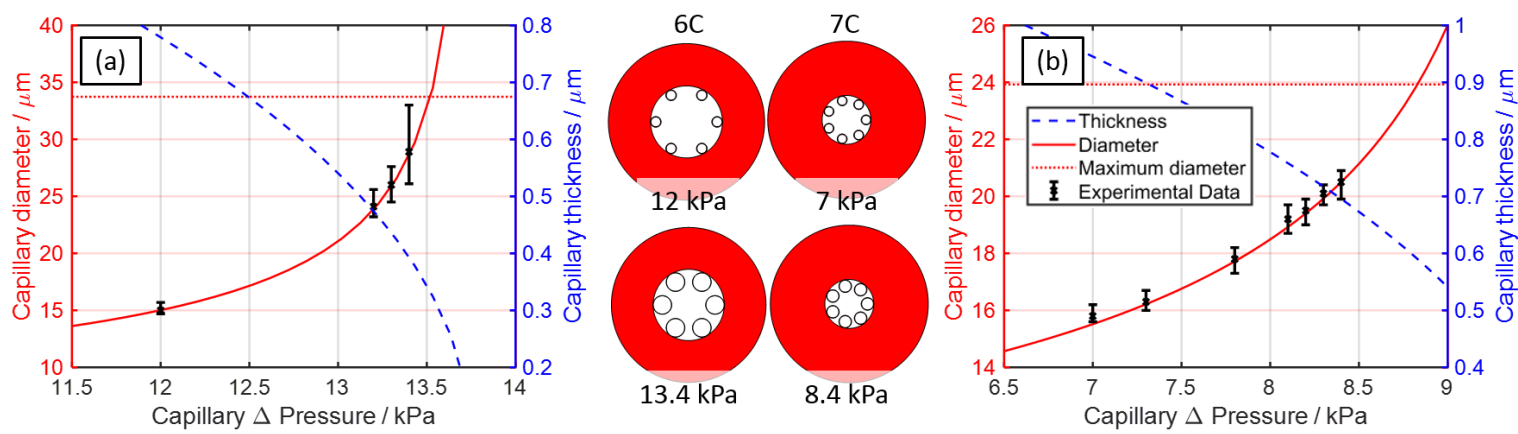

Fig. 2. Comparison of the simulated results and mean of the measured capillaries in the $6 \mathrm{C}$ fiber (a), and the 7C fiber (b). A constant pressure offset has been applied to the simulated data of (a): $1.35 \mathrm{kPa}$ and (b) $1.5 \mathrm{kPa}$. The error bars indicate the range of measured values, the maximum diameter, when the capillaries touch, is also indicated. Simulated fiber geometries are shown at the specified pressures.

The simulations match the experiments extremely well, demonstrating that this simple model is an effective tool for predicting the fiber geometry and can be used to increase the productivity of an otherwise trial and error development cycle. However, the tool has a far greater strength: consider the enlarged range of capillary diameters (indicated by the error bars) and the much steeper gradient in the $6 \mathrm{C}$ compared with the $7 \mathrm{C}$. The $6 \mathrm{C}$ draw was much more sensitive to subtle irregularities (either in preform or draw conditions) which resulted in a range of capillary diameters but also a range of thicknesses $350-450 \mathrm{~nm}$ which degrade its optical performance. The $7 \mathrm{C}$ configuration in contrast was extremely stable, allowing the production of a low loss geometry, as demonstrated in [5]. Several factors contribute to this improved stability, but the most significant is that the $7 \mathrm{C}$ fiber requires smaller capillaries and as such a reduced expansion compared with the $6 \mathrm{C}$. This keeps the gradient of the diameter curves small and the resulting structure more uniform. These tubular fiber designs appear simple but there are free parameters requiring design decisions. When upscaling the fiber yield such parameters need careful consideration as upscaling implicitly increases expansion leading to increased sensitivity. This model can explore those parameters to identify optimum preform geometry and draw conditions offering the best route to stable, uniform, low loss fiber.

The authors acknowledge the support of the Royal Academy of Engineering, ERC (grant agreement no. 682724), EPSRC programme grant Airguide Photonics (EP/P030181/1), and the Royal Society.

[1] N. V. Wheeler, T. D. Bradley, J. R. Hayes, M. A. Gouveia, S. Liang, Y. Chen, S. R. Sandoghchi, S. M. Abokhamis Mousavi, F. Poletti, M. N. Petrovich, and D. J. Richardson, "Low-loss Kagome hollow-core fibers operating from the near- to the mid-IR," Opt. Lett. 42, 2571-2574 (2017).

[2] Francesco Poletti, "Nested antiresonant nodeless hollow core fiber," Opt. Express 22, 23807-23828 (2014).

[3] Walter Belardi and Jonathan C. Knight, "Hollow antiresonant fibers with reduced attenuation," Opt. Lett. 39, 1853-1856 (2014).

[4] A. F. Kosolapov, G. K. Alagashev, A. N. Kolyadin, A. D. Pryamikov, A. S. Biryukov, I. A. Bufetov and E. M. Dianov. "Hollow-core revolver fibre with a double-capillary reflective cladding." Quantum Electronics 46, no. 3, 267 (2016).

[5] J. R. Hayes, S. R. Sandoghchi, T. D. Bradley, Z. Liu, R. Slavík, M. A. Gouveia, N. V. Wheeler, G. Jasion, Y. Chen, E. N. Fokoua, M. N.

Petrovich, D. J. Richardson, and F. Poletti, "Antiresonant Hollow Core Fiber With an Octave Spanning Bandwidth for Short Haul Data Communications," J. Lightwave Technol. 35, 437-442 (2017).

[6] A. D. Fitt, K. Furusawa, T. M. Monro, C. P. Please, and D. J. Richardson. "The mathematical modelling of capillary drawing for holey fibre manufacture." J. of Eng. Mathematics 43, no. 2-4, 201-227, (2002).

[7] G. T. Jasion, J. S. Shrimpton, Y. Chen, T. Bradley, D. J. Richardson, and F. Poletti, "MicroStructure Element Method (MSEM): viscous flow model for the virtual draw of microstructured optical fibers," Opt. Express 23, 312-329 (2015). 